\title{
Identifying Riemannian Singularities with Regular Non-Riemannian Geometry
}

\author{
Kevin Morand $\odot^{*}$ and Jeong-Hyuck Park $\odot^{\dagger}$ \\ Department of Physics, Sogang University, 35 Baekbeom-ro, Mapo-gu, Seoul 04107, Korea \\ Miok Park $\oplus^{\ddagger}$ \\ Center for Theoretical Physics of the Universe, Institute for Basic Science, Daejeon 34126, Korea \\ and School of Physics, Korea Institute for Advanced Study, Seoul 02455, Korea
}

(Received 8 June 2021; accepted 24 November 2021; published 26 January 2022)

\begin{abstract}
Admitting non-Riemannian geometries, double field theory extends the notion of spacetime beyond the Riemannian paradigm. We identify a class of singular spacetimes known in general relativity with regular non-Riemannian geometries. The former divergences merely correspond to coordinate singularities of the generalized metric for the latter. Computed in the string frame, they feature an impenetrable nonRiemannian sphere outside of which geodesics are complete with no singular deviation. Approaching the non-Riemannian points, particles freeze and strings become (anti)chiral.
\end{abstract}

DOI: 10.1103/PhysRevLett.128.041602

Introduction.-Spacetime singularities urge general relativity (GR) to evolve. If not in — still elusive - quantum gravity, the singularities in any Riemannian metric-based classical theories of gravity have several layers: (i) curable, coordinate singularity of the metric, (ii) genuine, curvature singularity, and (iii) geodesic incompleteness, as featured prominently in Penrose's theorem [1]. Here the item label (i), e.g., reflects the undoubled spacetime case, and later the label (i)) reflects the doubled spacetime case. However, on the one hand, (ii) does not necessarily imply (iii) (see, e.g., [2] for a recent example), and, on the other hand, one encounters an intrinsic ambiguity in choosing a frame $[3,4]$ when applying these notions to scalartensor theories (e.g., [5]), since Weyl transformations simply do not leave curvatures and geodesics invariant.

From a string theory perspective, the Riemannian metric $g_{\mu \nu}$ is only a fraction of the massless sector of closed strings which should further contain a skew-symmetric $B$-field and a scalar dilaton $\phi$. The theory implies a crucial symmetry, $\mathbf{O}(D, D)$ with spacetime dimension $D$, which transforms the trio $\{g, B, \phi\}$ into one another [6,7]. It calls for an $\mathbf{O}(D, D)$ singlet, $e^{-2 d}=\sqrt{-g} e^{-2 \phi}$, and an $\mathbf{O}(D, D)$ tensor,

$$
\begin{aligned}
\mathcal{H}_{A B} & =\left(\begin{array}{cc}
g^{-1} & -g^{-1} B \\
B g^{-1} & g-B g^{-1} B
\end{array}\right) \\
& =\left(\begin{array}{ll}
1 & 0 \\
B & 1
\end{array}\right)\left(\begin{array}{cc}
g^{-1} & 0 \\
0 & g
\end{array}\right)\left(\begin{array}{cc}
1 & -B \\
0 & 1
\end{array}\right),
\end{aligned}
$$

Published by the American Physical Society under the terms of the Creative Commons Attribution 4.0 International license. Further distribution of this work must maintain attribution to the author(s) and the published article's title, journal citation, and DOI. Funded by SCOAP ${ }^{3}$. which served as a generalized metric in the $\mathbf{O}(D, D)$ manifest formulations of both string worldsheet actions [8-16] and target spacetime effective descriptions currently called double field theory (DFT) [17-22]. By taking not $\{g, B, \phi\}$ but the $\mathbf{O}(D, D)$ multiplets themselves as the fundamental variables, DFT opens a new avenue beyond the Riemannian paradigm [23-26]. In this approach, $e^{-2 d}$ is an elementary scalar density with a unit diffeomorphic weight, and $\mathcal{H}_{A B}$ satisfies its own defining properties:

$$
\mathcal{H}_{A B}=\mathcal{H}_{B A}, \quad \mathcal{H}_{A}{ }^{C} \mathcal{H}_{B}{ }^{D} \mathcal{J}_{C D}=\mathcal{J}_{A B} .
$$

Here,

$$
\mathcal{J}_{A B}=\left(\begin{array}{ll}
\mathbf{0} & \mathbf{1} \\
\mathbf{1} & \mathbf{0}
\end{array}\right)
$$

is the $\mathbf{O}(D, D)$ invariant metric which, with its inverse, lowers and raises the $\mathbf{O}(D, D)$ indices, $A, B=1,2, \ldots, 2 D$. Seen as a square matrix, $\mathcal{H}_{A}{ }^{B}$ squares to identity and, hence, is invertible with $|\operatorname{det} \mathcal{H}|=1$.

Remarkably, DFT can be formulated in terms of any generalized metric that satisfies the defining relations (2) and may have evolved to an alternative "pure gravity" in the sense that both $d$ and $\mathcal{H}_{A B}$, i.e., the whole closed string massless sector, are taken as the fundamental, gravitational fields. It is by now fully equipped with its own Christoffel symbols $\Gamma_{A B C}$, scalar/Ricci/Einstein curvatures [27,28], and Einstein equations coupled to extra "matter": $G_{A B}=$ $8 \pi G T_{A B}$ [29]. This single expression unifies the equations of motion of the fundamental variables $\left\{d, \mathcal{H}_{A B}\right\}$. Besides, as solutions to condition (2), all possible classical geometries which DFT is capable of describing have been classified by two non-negative integers, $(n, \bar{n})$ [24]. Only the type $(0,0)$ is Riemannian, amounting to the well-known 
parametrization (1), while others are non-Riemannian in nature: The upper left $D \times D$ block of the generalized metric is degenerate with "nullity," i.e., dimension of the kernel, $n+\bar{n}$, and, thus, does not yield an invertible Riemannian metric. For a non-Riemannian geometry to be a consistent string background at the quantum level, it turns out necessary to put $n=\bar{n}$ in the usual critical dimensions, $D=10$ or 26 [26]. Nonrelativistic string [30-32] or torsional/stringy Newton-Cartan gravities of recent interest [33-40] are of the type $(1,1)[25,41-47]$. The condition $n=\bar{n}$ further makes the non-Riemannian geometry compatible with type II supersymmetric DFT and superstring [42,48] (cf. Refs. [49,50]): For spin group $\operatorname{Spin}(t, s) \times \operatorname{Spin}(s, t)$ with $t+s=10$, the allowed range of $n=\bar{n}$ is from zero to $\min (t, s)$ [24].

Formally, DFT employs a doubled coordinate system, $x^{A}=\left(\tilde{x}_{\mu}, x^{\nu}\right)$, and subsequently unifies diffeomorphisms and $B$-field gauge symmetry into "doubled" diffeomorphisms. Yet, the geometry is not truly doubled: Half of the coordinate dependency should be turned off, e.g., by setting $\left(\partial / \partial \tilde{x}_{\mu}\right) \equiv 0$, up to $\mathbf{O}(D, D)$ rotations. This may suggest that half of the doubled coordinates are actually gauged [51]. By gauging, e.g., $\tilde{x}_{\mu}$ explicitly as $D x^{A}=\left(d \tilde{x}_{\mu}-A_{\mu}, d x^{\nu}\right)$, it is possible to define an $\mathbf{O}(D, D)$-symmetric proper length [52] by means of the corresponding pullback of the generalized metric $D x^{A} D x^{B} \mathcal{H}_{A B}$ and to further construct "doubled-yetgauged" actions for particles [53,54] and strings [23,42]. When the generalized metric is $(0,0)$ Riemannian, all the components of the auxiliary gauge connection $A_{\mu}$ appear quadratically in the actions. After integrating them out, one recovers the conventional particle and string actions. On the other hand, when the background is non-Riemannian, $n+\bar{n}$ components come out linearly to play the role of Lagrange multipliers. Consequently, the particle is frozen with identically vanishing proper velocities over the nonRiemannian $(n+\bar{n})$-dimensions. Furthermore, the string becomes chiral over the $n$-directions and antichiral over the $\bar{n}$-directions [24], as happens in the $(1,1)$ nonrelativistic string case [30]. This also implies that, at the classical level, chiral strings get frozen, too [26].

From the Riemannian perspective, all the nonRiemannian backgrounds are singular geometries, as the would-be Riemannian metric diverges. Contradistinctly, they are well-behaved regular geometries in the doubled framework. This motivates us to revisit known singular Riemannian geometries and examine their non-Riemannian regularity; cf. earlier related discussions in Refs. [53,55-57]. In this Letter, we report that a class of curvature singularities in GR are regular non-Riemannian geometries of DFT. They are at worst coordinate singularities of DFT. Moreover, we show through examples that the ordinary (undoubled) geodesics defined in their string frame-which descend from $\mathbf{O}(D, D)$-symmetric (doubled) geodesics-are complete and that physically measurable tidal forces do not diverge, in spite of Riemannian curvature singularities. This last statement, although motivated by the DFT perspective, holds independently within the conventional GR setup.

Main idea and results.-The Riemann-wise singular geometries of our interest assume the following generic form, with $x^{\mu}=\left(t, y, x^{i}\right)$ :

$$
\begin{aligned}
d s^{2} & =\frac{1}{F}\left(-d t^{2}+d y^{2}\right)+G_{i j} d x^{i} d x^{j}, \\
B_{(2)} & = \pm \frac{1}{F} d t \wedge d y+\frac{1}{2} \beta_{\mu \nu} d x^{\mu} \wedge d x^{\nu}, \\
e^{-2 \phi} & =F \Psi .
\end{aligned}
$$

Our nonexhaustive list of examples includes (a) $D=2$ black hole by Witten [58], (b) $D=4$ spherical solution by Burgess, Myers, and Quevedo [59], and (c) $D=10$ black 5-brane by Horowitz and Strominger [60]. All of them feature curvature singularities where $F$ vanishes, while $G_{i j}, \beta_{\mu \nu}, \Psi$ remain harmlessly regular. Substituting (3) into (1) yields the crucial observation (cf. Refs. [23,43,44,61] for earlier examples) that the coordinate singularity is absent in the $\mathbf{O}(D, D)$ fundamental variables-no negative power of $F$ appears:

$$
e^{-2 d}=\Psi \sqrt{G}, \quad \mathcal{H}=\left(\begin{array}{cc}
\mathbf{1} & \mathbf{0} \\
\boldsymbol{\beta} & \mathbf{1}
\end{array}\right) \stackrel{\circ}{\mathcal{H}}\left(\begin{array}{cc}
\mathbf{1} & -\beta \\
\mathbf{0} & \mathbf{1}
\end{array}\right),
$$

where, with Pauli matrices,

$$
\stackrel{\circ}{\mathcal{H}}_{A B}=\left(\begin{array}{cccc}
-F \sigma_{3} & 0 & \pm \sigma_{1} & 0 \\
0 & G^{-1} & 0 & 0 \\
\pm \sigma_{1} & 0 & 0 & 0 \\
0 & 0 & 0 & G
\end{array}\right)
$$

Clearly, at the points where $F=0$, the DFT geometry is non-Riemannian, specifically of type $(1,1)$, as expected from the underlying Minkowskian spin group signature.

We now revisit the three aforementioned layers of singularity from the "doubled" perspective.

(i)) Removing the coordinate singularity from generalized metric through doubled diffeomorphisms. - The singular term in $B_{(2)}$ is crucial in regularizing $\mathcal{H}$. In fact, in the examples below, this term being pure gauge would not be present when writing the solutions in their simplest form. We shall nevertheless introduce such a singular pure gauge term in order to match the privileged doubled coordinate system (3). Namely, through doubled diffeomorphisms, one can eliminate what should be construed as a coordinate singularity of the generalized metric.

(ii)) All the $\mathbf{O}(D, D)$-symmetric curvatures are regular.-Once the DFT fundamental variables $\left\{\mathcal{H}_{A B}, e^{-2 d}\right\}$ are made free of any singularity (and twice continuously differentiable), all the $\mathbf{O}(D, D)$-symmetric curvatures are automatically regular. In fact, all our examples satisfy the 
equations of motion of the NS-NS supergravity, possibly with an $\mathbf{O}(D, D)$-symmetric "cosmological constant":

$$
\begin{aligned}
R+4 \square \phi-4 \partial_{\mu} \phi \partial^{\mu} \phi-\frac{1}{12} H_{\mu \nu \rho} H^{\mu \nu \rho}-2 \Lambda_{\mathrm{DFT}} & =0, \\
R_{\mu \nu}+2 \nabla_{\mu}\left(\partial_{\nu} \phi\right)-\frac{1}{4} H_{\mu \rho \sigma} H_{\nu}{ }^{\rho \sigma} & =0, \\
d \star\left(e^{-2 \phi} H_{(3)}\right) & =0 .
\end{aligned}
$$

This readily implies that the corresponding nonsingular DFT ansatz (4) solves the DFT Einstein equations $G_{A B}+$ $\mathcal{J}_{A B} \Lambda_{\mathrm{DFT}}=0$ everywhere even at the non-Riemannian points of $F=0$. It also means that all the $\mathbf{O}(D, D)$ symmetric DFT curvatures are trivially regular [57]. Similarly, while the conventional dilaton $\phi$ diverges as $F \rightarrow 0$, the DFT dilaton $d$ remains finite [cf. (4)], and, hence, so does the $\mathbf{O}(D, D)$-symmetric version of the Fradkin-Tseytlin term [62] in doubled string actions [16,63].

(iii)) Geodesics are complete in the string frame: Impenetrable non-Riemannian sphere.-The fact that the DFT dilaton $e^{-2 d}$ carries a nontrivial diffeomorphic weight prevents it from coupling to the $\mathbf{O}(D, D)$-symmetric doubled-yet-gauged particle and string actions [23,53] and, hence, to particle and stringy geodesics, whose equations read, respectively [24,64],

$$
\begin{aligned}
e \frac{d}{d \tau}\left(e^{-1} \mathcal{H}_{A B} D_{\tau} x^{B}\right)+2 \Gamma_{A B C}\left(\bar{P} D_{\tau} x\right)^{B}\left(P D_{\tau} x\right)^{C} & =0, \\
\frac{1}{\sqrt{-h}} \partial_{\alpha}\left(\sqrt{-h} \mathcal{H}_{A B} D^{\alpha} x^{B}\right)+\Gamma_{A B C}\left(\bar{P} D_{\alpha} x\right)^{B}\left(P D^{\alpha} x\right)^{C} & =0,
\end{aligned}
$$

where $e$ and $h_{\alpha \beta}$ are the worldline einbein and worldsheet metric, respectively, while $P_{A B}, \bar{P}_{A B}$ stand for $\frac{1}{2}\left(\mathcal{J}_{A B} \pm\right.$ $\left.\mathcal{H}_{A B}\right)$ and $D_{\tau} x, D_{\alpha} x$ are the pullbacks of the doubled-yetgauged differential. This rigidity naturally settles the issue of the frame ambiguity. Upon the Riemannian background of (1), an $\mathbf{O}(D, D)$-symmetric particle follows geodesics defined in the string frame [65]: After fixing gauges and solving for the auxiliary connection, (7) can be shown to reproduce the standard (undoubled) expressions

$$
\begin{array}{r}
\dot{x}^{\mu}+\gamma_{\nu \rho}^{\mu} \dot{x}^{\nu} \dot{x}^{\rho}=0 \Leftrightarrow \frac{d}{d \lambda}\left(g_{\mu \nu} \dot{x}^{\nu}\right)-\frac{1}{2} \partial_{\mu} g_{\nu \rho} \dot{x}^{\nu} \dot{x}^{\rho}=0, \\
\partial_{+}\left(g_{\mu \nu} \partial_{-} x^{\nu}\right)+\partial_{-}\left(g_{\mu \nu} \partial_{+} x^{\nu}\right)+\left(H_{\mu \nu \rho}-\partial_{\mu} g_{\nu \rho}\right) \partial_{+} x^{\nu} \partial_{-} x^{\rho}=0 .
\end{array}
$$

Focusing on the ansatz (3) and by analogy with (ii)), the doubled formulation (7) in terms of the generalized metric $\mathcal{H}_{A B}$ may still suggest that geodesics are regular in the limit $F \rightarrow 0$, despite the fact that its undoubled counterpart (8) involves the singular Riemannian metric $g_{\mu \nu}$. Indeed, as we show below for the aforementioned three examples, both null and timelike geodesics are complete (at least) in the region $F>0$. The non-Riemannian points of $F=0$ form a sphere for $D>2$ (or hyperbola for $D=2$ ) inside of which $(F<0)$ no timelike or null geodesics can penetrate. Timelike and nonradial null geodesics cannot even come close to the sphere from outside $(F>0)$. Only radial null ones may approach the sphere with identically vanishing proper velocities taking infinite affine parameter. Besides, undoubled strings - as an alternative probe of the "singular" geometries [66,67]—become (anti)chiral: With light-cone coordinates $y^{ \pm}=y \pm t$ and also $\partial_{ \pm}=\left(\partial / \partial \sigma^{ \pm}\right)$on the worldsheet, as already used in (8), we get $\partial_{-} y^{+}=0=$ $\partial_{+} y^{-}\left(\right.$or $\partial_{+} y^{+}=0=\partial_{-} y^{-}$) in the limit $F \rightarrow 0$. Thus, the undoubled, or conventional, particle geodesics and string propagations agree with the non-Riemannian (freezing/ chiral/antichiral) behaviors predicted from the previous doubled-yet-gauged sigma model approach $[23,24]$ which relied on the auxiliary gauge connection as Lagrange multiplier (cf. the introduction).

(ii) and (iii) again: Geodesic deviations are also regular.-In DFT, there is no $\mathbf{O}(D, D)$-symmetric completion of the Riemann curvature $[27,68,69]$. Hence, the criterion of " $\mathbf{O}(D, D)$-symmetric curvature singularity" may appear unbalanced or somewhat unfair compared to GR. As a step toward restoring the balance as well as focusing on genuine physical quantities, we further analyze the geodesic deviation and the "tidal force" therein (again in the string frame):

$$
\frac{D^{2} \xi^{\mu}}{d \lambda^{2}}=R_{\nu \rho \sigma}^{\mu} \dot{x}^{\nu} \dot{x}^{\rho} \xi^{\sigma} .
$$

As the geodesics are complete and smooth for $F \geq 0$, their deviations $\xi^{\mu}$ should be regular. Then, although the Riemann curvature itself diverges, its contraction with the vanishing velocities $R^{\mu}{ }_{\nu \rho \sigma} \dot{x}^{\nu} \dot{x}^{\rho}$ as well as the square norm $\left|D^{2} \xi / d \lambda^{2}\right|^{2}=g_{\mu \nu}\left(D^{2} \xi^{\mu} / d \lambda^{2}\right)\left(D^{2} \xi^{\nu} / d \lambda^{2}\right)$ can be finite, thus preventing the physically measurable quantities from being singular. This further property will be checked to hold in the following section for examples (a)-(c).

Examples: Geodesics and string propagation.-We now take a closer look at each example [58-60] to analyze generic geodesics (8) supplemented by the Hamiltonian or Virasoro constraints

$g_{\mu \nu} \dot{x}^{\mu} \dot{x}^{\nu}=\mathcal{E}, \quad \partial_{+} x^{\mu} \partial_{+} x^{\nu} g_{\mu \nu}=0=\partial_{-} x^{\mu} \partial_{-} x^{\nu} g_{\mu \nu}$.

Hereafter, $\mathcal{E}$ is to be either -1 (timelike) or 0 (null).

(a) $D=2$ black hole: Non-Riemannian hyperbola.

The $D=2$ geometry à $l a$ Witten [58] is characterized by $d s^{2}=d y^{+} d y^{-} / F$ with $F=-1+y^{+} y^{-} / l^{2}=(F /|F|) e^{-2 \phi}$. It solves (6) when $\Lambda_{\mathrm{DFT}}=-\left(2 / l^{2}\right)$. From the on-shell value of the Ricci scalar $R=-\left(4 / l^{2} F\right)$, the hyperbola $y^{+} y^{-}=l^{2}$ corresponds to a curvature singularity. We stress that, though the $H$-flux is trivial in two dimensions, the $B$-field still plays a crucial role in making the generalized metric free of singularity (5). 
Since the metric is invariant under scaling $\delta y^{ \pm}= \pm y^{ \pm}$, there are two constants of motion for every geodesic:

$$
L=\left(y^{-} \dot{y}^{+}-y^{+} \dot{y}^{-}\right) / F, \quad \mathcal{E}=\dot{y}^{+} \dot{y}^{-} / F,
$$

which give, setting $\omega \equiv l \dot{y}^{+} / y^{+}$,

$$
\begin{aligned}
l \frac{d F}{d \lambda} & = \pm \sqrt{F\left[4 \mathcal{E}+\left(\frac{L^{2}}{l^{2}}+4 \mathcal{E}\right) F\right]}, \\
l^{2} \frac{d^{2} F}{d \lambda^{2}} & =\left(\frac{L^{2}}{l^{2}}+4 \mathcal{E}\right) F+2 \mathcal{E}, \\
\frac{L^{2}}{l^{2}}+4 \mathcal{E} & =\left(\omega-\frac{\mathcal{E}}{\omega}\right)^{2}+\left(\frac{\omega}{F}\right)^{2}+\frac{2\left(\omega^{2}-\mathcal{E}\right)}{F} .
\end{aligned}
$$

It follows that timelike geodesics $(\mathcal{E}=-1)$ starting from the region $F>0$ will never reach the non-Riemannian hyperbola, satisfying $F \geq 4 /\left[(L / l)^{2}-4\right]>0$. Null ones $(\mathcal{E}=0)$ may approach only at past or future infinity as $F=e^{ \pm L \lambda / l^{2}} F_{0}$, since the most general null geodesics are, from (11) with initial values $y_{0}^{ \pm}$and $F_{0}$ at $\lambda=0$, either

$$
\left(\begin{array}{c}
y^{+}(\lambda) \\
y^{-}(\lambda)
\end{array}\right)=\left(\begin{array}{c}
l^{2} / y_{0}^{-}+\left(y_{0}^{+}-l^{2} / y_{0}^{-}\right) e^{L \lambda / l^{2}} \\
y_{0}^{-}
\end{array}\right)
$$

or $y^{+}(\lambda)=y_{0}^{+}, \quad y^{-}(\lambda)=l^{2} / y_{0}^{+}+\left(y_{0}^{-}-l^{2} / y_{0}^{+}\right) e^{-L \lambda / l^{2}}$. For these two solutions, the only nonvanishing components in (9) are, respectively, $R^{+}{ }_{\nu \rho-} \dot{x}^{\nu} \dot{x}^{\rho}=-\left(L / y_{0}^{-} l\right)^{2}$ and $R^{-}{ }_{\nu \rho+} \dot{x}^{\nu} \dot{x}^{\rho}=-\left(L / y_{0}^{+} l\right)^{2}$, which are all finite with $\left|\left(D^{2} \xi / d \lambda^{2}\right)\right|^{2}=0$. In fact, the variations of the general solutions (12) by the free parameters, $y_{0}^{ \pm}$, also lead to finite deviation vectors $\xi^{\mu}$. We conclude that the space $F>0$ is geodesically complete with no singular deviation. We turn to the string dynamics (8) and (10), which read

$$
\begin{array}{ll}
\partial_{+} \partial_{-} y^{+}-\partial_{+} y^{+} \partial_{-} y^{+} \frac{\partial \ln F}{\partial y^{+}}=0, & \partial_{+} y^{+} \partial_{+} y^{-}=0, \\
\partial_{+} \partial_{-} y^{-}-\partial_{+} y^{-} \partial_{-} y^{-} \frac{\partial \ln F}{\partial y^{-}}=0, & \partial_{-} y^{+} \partial_{-} y^{-}=0 .
\end{array}
$$

The second relation gives either $\partial_{+} y^{+}=0$ or $\partial_{+} y^{-}=0$. If $\partial_{+} y^{+}=0$, the third implies $\partial_{+}\left[(1 / F) \partial_{-} y^{-}\right]=0$ such that $\partial_{-} y^{-}=F \mathbf{f}\left(\sigma^{-}\right)$for some one-variable function $\mathbf{f}\left(\sigma^{-}\right)$. Thus, $\partial_{-} y^{-}$vanishes when $F=0$. On the other hand, if $\partial_{+} y^{-}=0$, the first implies $\partial_{+}\left[(1 / F) \partial_{-} y^{+}\right]=0$ and $\partial_{-} y^{+}=F \mathbf{f}\left(\sigma^{-}\right)$. Thus, $\partial_{-} y^{+}$vanishes when $F=0$. Similar analysis holds for the last relation. We conclude that, in any case, one of $\left\{y^{+}, y^{-}\right\}$is chiral and the other is antichiral on the non-Riemannian hyperbola.

(b) $D=4$ two-parameter family of spherical solution. Our $D=4$ example is a spherical solution from Ref. [59]:

$$
\begin{aligned}
& d s^{2}=\frac{1}{F(r)}\left(-d t^{2}+d r^{2}\right)+\mathcal{R}(r)^{2}\left(d \vartheta^{2}+\sin ^{2} \vartheta d \varphi^{2}\right), \\
& B_{(2)}= \pm \frac{1}{F(r)} d t \wedge d r+h \cos \vartheta d t \wedge d \varphi,
\end{aligned}
$$

where $F(r)$ and $\mathcal{R}(r)^{2}$ are, with two free parameters $b$ and $h$ [53] (see also [29] for their physical interpretations),

$$
\begin{aligned}
\frac{1}{F(r)} & =\frac{1+\sqrt{1-h^{2} / b^{2}}}{2}\left(\frac{r}{r+b}\right)+\frac{1-\sqrt{1-h^{2} / b^{2}}}{2}\left(\frac{r+b}{r}\right), \\
\mathcal{R}(r)^{2} & =\left(r+\frac{1}{2} b-\frac{1}{2} \sqrt{b^{2}-h^{2}}\right)^{2}+\frac{1}{4} h^{2}=\frac{r(r+b)}{F(r)} .
\end{aligned}
$$

We require $0<|h| \leq b$, such that the only singular source in the metric is at $r=0$ as $\lim _{r \rightarrow 0} F(r)=0$. The Ricci scalar diverges at $r=0$ as $R \simeq-\left[2 /\left(b-\sqrt{b^{2}-h^{2}}\right) r\right]$. Nevertheless, with nonsingular $\mathcal{R}(0)^{-2}$ due to $h \neq 0$, the generalized metric $\mathcal{H}_{A B}$, dilaton $e^{-2 d}=\mathcal{R}(r)^{2} \sin \vartheta$, and DFT curvatures are all finitely regular. The nonRiemannian points of $F=0$ form a 2-sphere with nontrivial proper area $4 \pi \mathcal{R}(0)^{2}=2 \pi b\left(b-\sqrt{b^{2}-h^{2}}\right)$.

For geodesics, without loss of generality, we put $\vartheta=\pi / 2$. The conserved energy $E$ and angular momentum $L_{\varphi}$ set

$$
\dot{t}=E F(r), \quad \dot{\varphi}=L_{\varphi} \mathcal{R}(r)^{-2} .
$$

In particular, $\dot{t}$ vanishes on the non-Riemannian sphere. The remaining radial motion reads

$0=\dot{r}^{2}+V(r), \quad V(r)=\left[-\mathcal{E}-E^{2} F(r)+\frac{L_{\varphi}^{2}}{\mathcal{R}(r)^{2}}\right] F(r)$.

From $\lim _{r \rightarrow 0} V=0$, it follows that $\dot{r}$ also gets trivial at $r=0$. In fact, since $\lim _{r \rightarrow 0} V^{\prime}(r)=4 L_{\varphi}^{2} /\left[b\left(b-\sqrt{b^{2}-h^{2}}\right)^{2}\right]-$ $\left[2 \mathcal{E} /\left(b-\sqrt{b^{2}-h^{2}}\right)\right]$ is positive for either $\mathcal{E}=-1$ or $\mathcal{E}=$ 0 with $L_{\varphi} \neq 0$, the corresponding potential is positive close to $r=0^{+}$. Thus, both the timelike and the nonradial null geodesics cannot come close to the sphere from outside. Only the radial null ones with $\mathcal{E}=0=L_{\varphi}$ may, but it takes infinite affine parameter as the integral of $d \lambda=(E F)^{-1} d r$ diverges logarithmically. These results can be all attributed to the repulsive gravitational force around the nonRiemannian sphere, while it is attractive for large $r \sim \mathcal{R}$ as $g_{t t} \sim-1+\left[\left(b \sqrt{1-h^{2} / b^{2}}\right) / \mathcal{R}\right]$.

For the radial null geodesics $\dot{t}=E F=|\dot{r}|$, we also confirm that the deviation (9) is finitely regular: The only nontrivial components of $R^{\mu}{ }_{\nu \rho \sigma} \dot{x}^{\nu} \dot{x}^{\rho}$ at $r=0$ take the values $\left[\left( \pm 2 E^{2}\right) /\left(b-\sqrt{b^{2}-h^{2}}\right)^{2}\right]$ for $\mu, \sigma$ being $t$ or $r$, with $\left|\left(D^{2} \xi / d \lambda^{2}\right)\right|^{2}=0$.

In the limit $r \rightarrow 0$ and, hence, $F \rightarrow 0$, the string dynamics (8) and (10) implies, with $y^{ \pm}=r \pm t$, 


$$
\begin{aligned}
\partial_{+} y^{+} \partial_{-} y^{+} F^{\prime}(0) & =0, & \partial_{+} y^{+} \partial_{+} y^{-}=0, \\
\partial_{+} y^{-} \partial_{-} y^{-} F^{\prime}(0) & =0, & \partial_{-} y^{+} \partial_{-} y^{-}=0,
\end{aligned}
$$

where $F^{\prime}(0)=\lim _{r \rightarrow 0} F^{\prime}(r)=\left[2 /\left(b-\sqrt{b^{2}-h^{2}}\right)\right]$ is nonvanishing. This confirms that one of $\left\{y^{+}, y^{-}\right\}$is chiral while the other is antichiral on the non-Riemannian sphere.

(c) $D=10$ black 5-brane. One particular black 5-brane geometry from Ref. [60] reads

$$
\begin{aligned}
d s^{2} & =\frac{-d t^{2}+d r^{2}}{F(r)}+r^{2} d \Omega_{3}^{2}+d \vec{x}^{2}, \\
F & =1-\left(r_{c} / r\right)^{2}=e^{-2 \phi} .
\end{aligned}
$$

The Ricci scalar diverges at both $r=0$ and $r=r_{c}$ as $R=-4 r_{c}^{4} /\left[r^{4}\left(r^{2}-r_{c}^{2}\right)\right]$. Though the $H$-flux is trivial, a pure gauge $B$-field should be introduced, as prescribed in (3). The generalized metric (5) is then non-Riemannian regular on the 3 -sphere of the radius $r=r_{c}$ but still singular at $r=0$. We shall see soon that the non-Riemannian sphere forms the boundary of a geodesically complete space of $F>0$ which excludes the dangerous point $r=0$.

The geodesic analysis is similar to example (b) and reduces to $\dot{t}=E F$ and $\dot{r}^{2}+V(r)=0$ with a potential involving non-negative constants $L_{\Omega}^{2}$ (total angular momentum) and $\vec{P}^{2}$ (extra momentum):

$$
V(r)=\left[-\mathcal{E}-E^{2} F(r)+\frac{L_{\Omega}^{2}}{r^{2}}+\vec{P}^{2}\right] F(r) .
$$

Since $V^{\prime}\left(r_{c}\right)=2\left(-\mathcal{E}+L_{\Omega}^{2} / r_{c}^{2}+\vec{P}^{2}\right) / r_{c}$ is positive for either $\mathcal{E}=-1$ or $\mathcal{E}=0$ with $L_{\Omega}^{2} / r_{c}^{2}+\vec{P}^{2} \neq 0$, and $V\left(r_{c}\right)$ vanishes, the corresponding potential is positive close to $r=r_{c}^{+}$. Thus, timelike and generic null geodesics cannot reach the non-Riemannian sphere. Only the radial null ones having $\dot{t}=E F=|\dot{r}|$ and $L_{\Omega}^{2}=0=\vec{P}^{2}$ can, albeit taking infinite affine parameter with vanishing proper velocities. Besides, the deviation (9) is regular: The only nontrivial values of $R_{\nu \rho \sigma}^{\mu} \dot{x}^{\nu} \dot{x}^{\rho}$ at $r=r_{c}$ are $\pm 2 E^{2} / r_{c}^{2}$, for $\mu, \sigma$ being $t$ or $r$, with $\left|\left(D^{2} \xi / d \lambda^{2}\right)\right|^{2}=0$.

In the limit $r \rightarrow r_{c}$ and, hence, $F \rightarrow 0$, the chirality relations of the previous example (15) still hold after replacing $F^{\prime}(0)$ by $F^{\prime}\left(r_{c}\right)=2 / r_{c}$.

Discussion.-We have shown that the curvature singularities featured in a large class of GR spacetimes (3) are mere artifacts of Riemannian geometry. In particular, we have noted the remarkable fact that physically measurable tidal forces do not diverge. Further examples include $D=10$ superstring [70,71] with negative tension $[43,44,56,72,73]$. The corresponding generalized metric was shown to be regular in Ref. [56].

Converted to the Einstein frame, $g_{\mu \nu}^{\mathbf{E}}=e^{4 \phi /(2-D)} g_{\mu \nu}$, the $D>2$ examples feature more severe curvature singularities and become geodesically incomplete (e.g., [74]) with singular deviations. $D=2$ DFT is essentially
Jackiw-Teitelboim gravity $[75,76]$ as $g_{\mu \nu}^{\mathbf{J T}}=e^{-2 \phi} g_{\mu \nu}$. Witten's solution is then mapped to flat spacetime.

Constant non-Riemannian backgrounds were shown in Ref. [46] to admit infinite-dimensional isometries. All of them might be realized as the asymptotic symmetries of the non-Riemannian spheres with large radius (as $F^{\prime} \rightarrow 0$ ). The associated, infinitely many, conserved charges then might store all the information of the ingoing and freezing null radial geodesics. We call for further studies.

We thank Gungwon Kang, Nakwoo Kim, Wontae Kim, and Dong-han Yeom for useful discussions as well as anonymous referees for helpful suggestions. This work is supported by Basic Science Research Program through the National Research Foundation of Korea (NRF) through Grants No. NRF-2016R1D1A1B01015196, No. NRF2018H1D3A1A01030137 (Brain Pool Program), No. NRF-2020R1A6A1A03047877 (Center for Quantum Space Time), and No. NRF-2021R1C1C1005037. M. P. is further supported by a KIAS Individual Grant (PG062002) at Korea Institute for Advanced Study and by the Institute for Basic Science (Grant No. IBS-R018-Y1).

*morand@sogang.ac.kr †park@sogang.ac.kr \$miokpark76@ibs.re.kr

[1] R. Penrose, Gravitational Collapse and Space-Time Singularities, Phys. Rev. Lett. 14, 57 (1965).

[2] G. J. Olmo, D. Rubiera-Garcia, and A. Sanchez-Puente, Geodesic completeness in a wormhole spacetime with horizons, Phys. Rev. D 92, 044047 (2015).

[3] V. Faraoni, E. Gunzig, and P. Nardone, Conformal transformations in classical gravitational theories and in cosmology, Fundam. Cosm. Phys. 20, 121 (1999), https://arxiv.org/ abs/gr-qc/9811047.

[4] V. Faraoni and S. Nadeau, (Pseudo)issue of the conformal frame revisited, Phys. Rev. D 75, 023501 (2007).

[5] G. W. Horndeski, Second-order scalar-tensor field equations in a four-dimensional space, Int. J. Theor. Phys. 10, 363 (1974).

[6] T. H. Buscher, A symmetry of the string background field equations, Phys. Lett. B 194, 59 (1987).

[7] T. H. Buscher, Path-integral derivation of quantum duality in nonlinear sigma-models, Phys. Lett. B 201, 466 (1988).

[8] A. Giveon, E. Rabinovici, and G. Veneziano, Duality in string background space, Nucl. Phys. B322, 167 (1989).

[9] M. J. Duff, Duality rotations in string theory, Nucl. Phys. B335, 610 (1990).

[10] A. A. Tseytlin, Duality symmetric formulation of string world sheet dynamics, Phys. Lett. B 242, 163 (1990).

[11] A. A. Tseytlin, Duality symmetric closed string theory and interacting chiral scalars, Nucl. Phys. B350, 395 (1991).

[12] M. Roček and E. P. Verlinde, Duality, quotients, and currents, Nucl. Phys. B373, 630 (1992).

[13] A. Giveon and M. Roček, Generalized duality in curved string backgrounds, Nucl. Phys. B380, 128 (1992). 
[14] C. M. Hull, A geometry for non-geometric string backgrounds, J. High Energy Phys. 10 (2005) 065.

[15] C. M. Hull, Global aspects of T-duality, gauged sigma models and T-folds, J. High Energy Phys. 10 (2007) 057.

[16] C. M. Hull, Doubled geometry and T-folds, J. High Energy Phys. 07 (2007) 080.

[17] W. Siegel, Two vierbein formalism for string inspired axionic gravity, Phys. Rev. D 47, 5453 (1993).

[18] W. Siegel, Superspace duality in low-energy superstrings, Phys. Rev. D 48, 2826 (1993).

[19] C. Hull and B. Zwiebach, Double field theory, J. High Energy Phys. 09 (2009) 099.

[20] C. Hull and B. Zwiebach, The gauge algebra of double field theory and Courant brackets, J. High Energy Phys. 09 (2009) 090.

[21] O. Hohm, C. Hull, and B. Zwiebach, Background independent action for double field theory, J. High Energy Phys. 07 (2010) 016.

[22] O. Hohm, C. Hull, and B. Zwiebach, Generalized metric formulation of double field theory, J. High Energy Phys. 08 (2010) 008.

[23] K. Lee and J.H. Park, Covariant action for a string in doubled yet gauged spacetime, Nucl. Phys. B880, 134 (2014).

[24] K. Morand and J. H. Park, Classification of non-Riemannian doubled-yet-gauged spacetime, Eur. Phys. J. C 77, 685 (2017).

[25] K. Cho and J. H. Park, Remarks on the non-Riemannian sector in Double Field Theory, Eur. Phys. J. C 80, 101 (2020).

[26] J. H. Park and S. Sugimoto, String Theory and nonRiemannian Geometry, Phys. Rev. Lett. 125, 211601 (2020).

[27] I. Jeon, K. Lee, and J. H. Park, Stringy differential geometry, beyond Riemann, Phys. Rev. D 84, 044022 (2011).

[28] J. H. Park, S. J. Rey, W. Rim, and Y. Sakatani, O(D, D) covariant Noether currents and global charges in double field theory, J. High Energy Phys. 11 (2015) 131.

[29] S. Angus, K. Cho, and J. H. Park, Einstein double field equations, Eur. Phys. J. C 78, 500 (2018).

[30] J. Gomis and H. Ooguri, Nonrelativistic closed string theory, J. Math. Phys. (N.Y.) 42, 3127 (2001).

[31] U. H. Danielsson, M. Kruczenski and A. Güijosa, IIA/B, wound and wrapped, J. High Energy Phys. 10 (2000) 020.

[32] J. Gomis, J. Gomis, and K. Kamimura, Non-relativistic superstrings: a new soluble sector of $\operatorname{AdS}(5) \times S^{5}$, J. High Energy Phys. 12 (2005) 024.

[33] M. H. Christensen, J. Hartong, N. A. Obers, and B. Rollier, Torsional Newton-Cartan geometry and Lifshitz holography, Phys. Rev. D 89, 061901 (2014).

[34] J. Hartong and N. A. Obers, Hořava-Lifshitz gravity from dynamical Newton-Cartan geometry, J. High Energy Phys. 07 (2015) 155.

[35] T. Harmark, J. Hartong, and N. A. Obers, Nonrelativistic strings and limits of the AdS/CFT correspondence, Phys. Rev. D 96, 086019 (2017).

[36] T. Harmark, J. Hartong, L. Menculini, N. A. Obers, and Z. Yan, Strings with non-relativistic conformal symmetry and limits of the AdS/CFT correspondence, J. High Energy Phys. 11 (2018) 190.
[37] E. Bergshoeff, J. Gomis, and Z. Yan, Nonrelativistic string theory and T-duality, J. High Energy Phys. 11 (2018) 133.

[38] E. A. Bergshoeff, J. Gomis, J. Rosseel, C. Şimşek, and Z. Yan, String theory and string Newton-Cartan geometry, J. Phys. A 53, 014001 (2019).

[39] T. Harmark, J. Hartong, L. Menculini, N. A. Obers, and G. Oling, Relating non-relativistic string theories, J. High Energy Phys. 11 (2019) 071.

[40] E. A. Bergshoeff, J. Lahnsteiner, L. Romano, J. Rosseel, and C. Şimşek, A non-relativistic limit of NS-NS gravity, J. High Energy Phys. 06 (2021) 021.

[41] S. M. Ko, C. Melby-Thompson, R. Meyer, and J. H. Park, Dynamics of perturbations in double field theory \& nonrelativistic string theory, J. High Energy Phys. 12 (2015) 144.

[42] J. H. Park, Green-Schwarz superstring on doubledyet-gauged spacetime, J. High Energy Phys. 11 (2016) 005.

[43] D. S. Berman, C.D. A. Blair, and R. Otsuki, NonRiemannian geometry of M-theory, J. High Energy Phys. 07 (2019) 175.

[44] C. D. A. Blair, A worldsheet supersymmetric NewtonCartan string, J. High Energy Phys. 10 (2019) 266.

[45] A. D. Gallegos, U. Gürsoy, S. Verma, and N. Zinnato, NonRiemannian gravity actions from double field theory, J. High Energy Phys. 06 (2021) 173.

[46] C. D. A. Blair, G. Oling, and J. H. Park, Non-Riemannian isometries from double field theory, J. High Energy Phys. 04 (2021) 072.

[47] C. D. A. Blair, D. Gallegos, and N. Zinnato, A nonrelativistic limit of M-theory and 11-dimensional membrane Newton-Cartan geometry, J. High Energy Phys. 10 (2021) 015 .

[48] I. Jeon, K. Lee, J. H. Park, and Y. Suh, Stringy unification of type IIA and IIB supergravities under $\mathcal{N}=2 \quad D=10$ supersymmetric double field theory, Phys. Lett. B 723, 245 (2013).

[49] A. Coimbra, C. Strickland-Constable, and D. Waldram, Supergravity as generalised geometry I: Type II theories, J. High Energy Phys. 11 (2011) 091.

[50] M. Hatsuda, K. Kamimura, and W. Siegel, Type II chiral affine Lie algebras and string actions in doubled space, J. High Energy Phys. 09 (2015) 113.

[51] J. H. Park, Comments on double field theory and diffeomorphisms, J. High Energy Phys. 06 (2013) 098.

[52] J. H. Park, Stringy Gravity: Solving the Dark Problems at 'short' distance, EPJ Web Conf. 168, 01010 (2018).

[53] S. M. Ko, J. H. Park, and M. Suh, The rotation curve of a point particle in stringy gravity, J. Cosmol. Astropart. Phys. 06 (2017) 002.

[54] T. Basile, E. Joung, and J. H. Park, A note on FaddeevPopov action for doubled-yet-gauged particle and graded Poisson geometry, J. High Energy Phys. 02 (2020) 022.

[55] E. Malek, Timelike U-dualities in generalised geometry, J. High Energy Phys. 11 (2013) 185.

[56] C. D. A. Blair, Doubled strings, negative strings and null waves, J. High Energy Phys. 11 (2016) 042.

[57] D. S. Berman and F. J. Rudolph, Strings, branes and the selfdual solutions of Exceptional Field Theory, J. High Energy Phys. 05 (2015) 130. 
[58] E. Witten, String theory and black holes, Phys. Rev. D 44, 314 (1991).

[59] C. P. Burgess, R. C. Myers, and F. Quevedo, On spherically symmetric string solutions in four-dimensions, Nucl. Phys. B442, 75 (1995).

[60] G. T. Horowitz and A. Strominger, Black strings and p-branes, Nucl. Phys. B360, 197 (1991).

[61] C. D. A. Blair, Conserved currents of double field theory, J. High Energy Phys. 04 (2016) 180.

[62] E. S. Fradkin and A. A. Tseytlin, Effective field theory from quantized strings, Phys. Lett. 158B, 316 (1985).

[63] J. J. Fernández-Melgarejo, J. I. Sakamoto, Y. Sakatani, and K. Yoshida, Weyl Invariance of String Theories in Generalized Supergravity Backgrounds, Phys. Rev. Lett. 122, 111602 (2019).

[64] The second formula in (7) holds for $(0,0)$ Riemannian cases where the self-duality $D_{\alpha} x^{A}+(1 / \sqrt{-h}) \epsilon_{\alpha}{ }^{\beta} \mathcal{H}^{A}{ }_{B} D_{\beta} x^{B}=0$ is valid for all $A$. For the general expression, see [23].

[65] Since the null energy condition can be broken by the term $2 \nabla_{\mu}\left(\partial_{\nu} \phi\right)$ in (6), Penrose's theorem can be evaded in the string frame, thus allowing for geodesically complete spaces.

[66] M. J. Duff, R. R. Khuri, and J. X. Lu, String and five-brane solitons: Singular or nonsingular? Nucl. Phys. B377, 281 (1992).
[67] M. J. Duff, R. R. Khuri, and J. X. Lu, String solitons, Phys. Rep. 259, 213 (1995).

[68] I. Jeon, K. Lee, and J. H. Park, Differential geometry with a projection: application to double field theory, J. High Energy Phys. 04 (2011) 014.

[69] O. Hohm and B. Zwiebach, On the Riemann tensor in double field theory, J. High Energy Phys. 05 (2012) 126.

[70] A. Dabholkar, G. W. Gibbons, J. A. Harvey, and F. Ruiz Ruiz, Superstrings and solitons, Nucl. Phys. B340, 33 (1990).

[71] J. Berkeley, D. S. Berman, and F. J. Rudolph, Strings and branes are waves, J. High Energy Phys. 06 (2014) 006.

[72] R. Dijkgraaf, B. Heidenreich, P. Jefferson, and C. Vafa, Negative branes, supergroups and the signature of spacetime, J. High Energy Phys. 02 (2018) 050.

[73] A. S. Arvanitakis and C. D. A. Blair, Black hole thermodynamics, stringy dualities and double field theory, Classical Quantum Gravity 34, 055001 (2017).

[74] S. Kang and D. h. Yeom, Causal structures and dynamics of black-hole-like solutions in string theory, Eur. Phys. J. C 79, 927 (2019).

[75] C. Teitelboim, Gravitation and hamiltonian structure in two spacetime dimensions, Phys. Lett. 126B, 41 (1983).

[76] R. Jackiw, Lower dimensional gravity, Nucl. Phys. B252, 343 (1985). 